\title{
MATRIX DIFFERENTIAL EQUATION AND HIGHER-ORDER NUMERICAL METHODS FOR PROBLEMS OF NON-LINEAR CREEP, VISCOELASTICITY AND ELASTO-PLASTICITY*
}

\author{
Z. P. BAŽANT $†$ \\ Northwestern University, Evanston, Illinois 60201
}

SUMMARY

The constitutive equation is assumed in a very general form which includes as special cases non-linear creep, incremental elasto-plasticity as well as viscoelasticity represented by a chain of $n$ standard solid models. Subdividing the structure into $N$ finite elements, the problem of structural analysis is formulated with a system of $6 N(n+1)$ ordinary non-linear first-order differential equations in terms of the components of stresses and strains in the elements. This formulation enables one to apply RungeKutta methods or the predictor-corrector methods.

\section{INTRODUCTION}

The problems of creep, elasto-plasticity or viscoelasticity represent initial-boundary value problems in space co-ordinates $x, y, z$ and time $t$. For integration in space co-ordinates, some approximate method, such as the finite element method, $, 1,2$ the finite difference method ${ }^{3}$ or various series expansions, may be adopted. In such an approach, the state of deformation or stress is characterized with a finite number of variables for which a system or ordinary differential equations may be obtained. The practical solution, however, has been carried out by a simple step-by-step method as a sequence of elasticity problems, ${ }^{3-11}$ without regard to any formulation by a system of ordinary differential equations. If a comparison with the well-known numerical methods for the initial-boundary value problem in ordinary differential equations ${ }^{12}$ is made, it may be found that the step-by-step methods used (eventually improved by iterations in each step ${ }^{9}$ ) correspond to the crudest type of numerical methods, the Euler method, which is actually almost never used for computer integration of ordinary differential equations because more powerful methods are available. These include, for example, the Runge-Kutta methods and the predictorcorrector methods. The generalized algorithm of these methods has so far been formulated only for the linear rate-type creep of ageing materials (concrete), ${ }^{13}$ and tested by a few analyses of inhomogeneous prestressed concrete structures.

\section{CONSTITUTUTIVE EQUATION}

Attention will be restricted to small deformations and the stress-strain relations will be considered under a very general form:

$$
\varepsilon=\sum_{\mu=1}^{n} \varepsilon_{\mu}, \quad \dot{\varepsilon}_{\mu}=\mathbf{A}_{\mu} \dot{\sigma}+\dot{\varepsilon}_{\mu}^{0} \quad(\mu=1, \ldots, n)
$$

\footnotetext{
* This paper is largely based on the author's Report No. 68/2, Approximate Analysis of Linear and Nonlinear Creep Problems, Department of Civil Engineering, University of Toronto, December, 1968, prepared under the sponsorship of Ford Science Foundation. Further refinement was obtained in connection with the preliminary research for the National Science Foundation project, Grant No. GK-26030.

$\dagger$ Associate Professor of Civil Engineering.
} 
where

$$
\dot{\boldsymbol{\varepsilon}}_{\mu}^{\mathbf{0}}=\widehat{\mathbf{C}}_{\mu}\left(\boldsymbol{\sigma}, \boldsymbol{\varepsilon}_{\mu}, t\right), \quad \mathbf{A}_{\mu}=\widehat{\mathbf{A}}_{\mu}\left(\boldsymbol{\sigma}, \boldsymbol{\varepsilon}_{\mu}, t\right)
$$

Here $\varepsilon=\left(\varepsilon_{x x}, \varepsilon_{y}, \varepsilon_{z}, \gamma_{x y}, \gamma_{y z}, \gamma_{z x}\right)^{\mathrm{T}}=$ column matrix formed of strain components in Cartesian axes $x, y, z$; superscript $\mathrm{T}$ stands here and in the sequel for the transpose of a matrix; $\varepsilon_{1}, \ldots, \varepsilon_{n}=$ column matrices of partial strains, of a form analogous to $\varepsilon ; \varepsilon_{1}^{0}, \ldots, \varepsilon_{n}^{0}$ are column matrices, analogous to $\varepsilon$, whose sum represents the column matrix of inelastic strains, $\varepsilon^{0} ; \boldsymbol{\sigma}=\left(\sigma_{x}, \sigma_{y}, \sigma_{z}, \tau_{x y}, \tau_{y z}, \tau_{z x}\right)^{\mathbf{T}}=$ column matrix formed of stress components; $\mathbf{A}_{1}, \ldots, \mathbf{A}_{n}$ square matrices $(6 \times 6)$ of the partial incremental elastic constants; $\hat{\mathbf{A}}_{\mu}$ is a square matrix function. and $\hat{\mathbf{C}}_{\mu}$ is a column matrix function of $\sigma, \varepsilon_{\mu}$ and time $t$. The function $\hat{\mathbf{C}}_{\mu}$ may contain terms independent of $\sigma$ and $\varepsilon_{\mu}$. Such terms represent the rate of thermal dilatation or shrinkage. The remaining terms represent the rates of creep strains. A dot as in $\dot{\boldsymbol{\varepsilon}}$ or $\dot{\boldsymbol{\sigma}}$ denotes the time rate which may be considered here as a partial derivative with respect to time $t$, e.g. $\dot{\boldsymbol{\varepsilon}}=\partial \boldsymbol{\varepsilon} / \partial t$. The matrices $\mathbf{A}_{\mu}$ and matrix functions $\hat{\mathbf{A}}_{\mu}$ and $\hat{\mathbf{C}}_{\mu}$ clearly cannot have arbitrary forms but must satisfy the wellknown invariance requirements and certain inequalities following from thermodynamics.

It should be noted that the constitutive equation (1) includes, for example, the following special cases:

1. The typical non-linear creep law of rate-type (such as the primary creep of metals or, approximately, creep of concrete at high stress) if $n=1, \hat{\mathbf{A}}_{1}$ is independent of $\sigma, \varepsilon$ and $t$, and $\hat{C}_{1}$ is independent of $t$.

2. The non-linear elastic material in small strain, which may approximate a strain-hardening elasto-plastic material (see Reference 1, equations 12.8, 9, 11, 14) during loading, provided the state of ideal plasticity has not been reached, so that both $\widehat{\mathbf{A}}_{\mu}$ and $\hat{\mathbf{A}}_{\mu}^{-1}$ exist. In this case $t=$ load parameter rather than time; $n=1, \widehat{\mathbf{C}}_{1}=0 ; \widehat{\mathbf{A}}_{1}$ depends only on $\sigma$ (including its second invariant).

3. The general linear viscoelastic material described by a chain of standard solid models (or Kelvin models), ${ }^{10}$ each of which is associated with one of the variables $\varepsilon_{1}, \ldots, \varepsilon_{n}$. This occurs if $\mathbf{D}_{\mu}-s$ are independent of $\boldsymbol{\sigma}, \boldsymbol{\varepsilon}, t$ and $\widehat{\mathbf{C}}_{\mu}-s$ are linear functions of $\boldsymbol{\sigma}, \boldsymbol{\varepsilon}$.

4. The standard solid body if, in addition to item $3, n=1$.

5. The linear creep of an ageing material, such as concrete, if the conditions are the same as in item 3, except that $\mathbf{D}_{\mu}-s$ and $\hat{\mathbf{C}}_{\mu}-s$ depend on $t$.

6. The creep law of concrete at variable water content and temperature, if $\mathbf{D}_{\mu}-s$ and $\hat{\mathbf{C}}_{\mu}-s$ depend on the solution of the independent problems of water diffusion, heat conduction and hydration reactions.

\section{FORMULATION IN TERMS OF ORDINARY DIFFERENTIAL EQUATIONS}

The finite element method in the displacement approach ${ }^{1,2}$ will now be considered. The given body is assumed to be subdivided into $N$ constant-strain elements (tetrahedera, for instance). Denote by $\mathbf{r}$ the column matrix of all displacement components in all nodes, and by $N$ the number of all components. It will be assumed that $\mathbf{r}$ does not contain the displacement components which are to be zero because of the given support conditions. The strains $\varepsilon^{(i)}$ in the $i$ th element are related to $\mathbf{r}$ as follows:

$$
\boldsymbol{\varepsilon}^{(i)}=\mathbf{b}^{(i)} \mathbf{r}
$$

where $\mathbf{b}^{(i)}$ is a rectangular matrix depending on co-ordinates alone, with 6 rows and $N$ columns, of which only the columns with the numbers of the displacement components of the nodes of the $i$ th element are non-zero.

Consider an infinitesimal time increment $\mathrm{d} t$, at the beginning of which the values $\sigma^{(i)}, \varepsilon_{1}^{(i)}, \ldots, \varepsilon_{n}^{(i)}$ of $\sigma, \varepsilon_{1}, \ldots, \varepsilon_{n}$ in each element are known. From equation (1) it follows that

$$
\mathrm{d} \boldsymbol{\sigma}^{(i)}=\mathbf{D}^{(i)}\left(\mathrm{d} \boldsymbol{\varepsilon}^{(i)}-\dot{\boldsymbol{\varepsilon}}^{(i)} \mathrm{d} t\right)
$$


where

$$
\mathbf{D}^{(i)}=\sum_{\mu} \mathbf{A}_{\mu}^{(i)-1}, \quad \boldsymbol{\varepsilon}^{(i)}=\sum_{\mu} \boldsymbol{\varepsilon}_{\mu}^{0(i)}
$$

Here $\mathbf{D}^{(i)}$ represents the matric of incremental elastic moduli. Because $\dot{\varepsilon}^{(0)} \mathrm{d} t$ can be determined, according to (1a), from the initial values of $\sigma^{(i)}, \varepsilon_{1}^{(i)}, \ldots, \varepsilon_{n}^{(i)}$ in the interval $\mathrm{d} t$, equation (3) may be regarded as an incremental elastic stress-strain law with prescribed initial strains $\dot{\boldsymbol{\varepsilon}}^{0(i)} \mathrm{d} t$.

To determine the equilibrium conditions for the infinitesimal increments dr, consider an arbitrary virtual displacement $\delta \mathbf{r}$, satisfying the boundary conditions of place. The virtual work done by the prescribed increments $\mathrm{dF}$ of the applied nodal forces $\mathbf{F}$ is

$$
(\delta \mathbf{r})^{\mathbf{T}} \mathrm{dF}
$$

The associated virtual strains in the $i$ th element are $\delta \varepsilon^{(i)}=\mathbf{b}^{(i)} \delta \mathbf{r}$. The virtual work of the stress increments is $\sum_{i}\left(\delta \varepsilon^{(i)}\right)^{\mathbf{T}} \mathrm{d} \sigma^{(i)} V^{(i)}$, which equals, according to equations (3) and (2).

$$
(\delta \mathbf{r})^{\mathrm{T}} \sum_{i=1}^{N}\left(\mathbf{b}^{(i)^{\mathrm{T}}} \mathbf{D}^{(i)} \mathbf{b}^{(i)} \mathrm{d} \mathbf{r}-\mathbf{b}^{(i)^{\mathrm{T}}} \mathbf{D}^{(i)} \dot{\boldsymbol{\varepsilon}}^{0(i)} \mathrm{d} t\right) V^{(i)}
$$

where $V^{(i)}$ is the volume of the $i$ th element. According to the principle of virtual displacements, expressions (4a) and (4b) must be equal for any $\delta \mathbf{r}$. This is verified if, and only if $\mathbf{K} \mathrm{d} \mathbf{r}-\dot{\mathbf{F}}^{0} \mathrm{~d} t=\mathrm{d} \mathbf{F}$ or

where

$$
\dot{\mathbf{r}}=\mathbf{K}^{-1}\left(\dot{\mathbf{F}}+\dot{\mathbf{F}}^{0}\right)
$$

$$
\begin{gathered}
\mathbf{K}=\sum_{i=1}^{N} \mathbf{b}^{(i)^{\mathbf{T}}} \mathbf{D}^{(i)} \mathbf{b}^{(i)} V^{(i)} \\
\dot{\mathbf{F}}^{0}=\sum_{i=1}^{N} \mathbf{b}^{(i)^{\mathrm{T}}} \mathbf{D}^{(i)} \dot{\varepsilon}^{0(i)} V^{(i)}
\end{gathered}
$$

Here $\mathbf{K}$ represents the incremental stiffness matrix (of size $N \times N$ ), which is non-singular as long as the structure is stable, and $\mathbf{F}^{0}$ is a column matrix of nodal force rates equivalent to inelastic strains.

Unlike in an elastic problem, the system of differential equations (5) alone is insufficient for solving $\mathbf{r}$, and must be completed by equations (1), written for each element. If equations (5) are combined with (2), and $\mathbf{K}, \dot{\mathbf{F}}^{0}$ are expressed with the help of equations (6a, b), (3a) and (1a), the following equations can be obtained:

$$
\begin{aligned}
\dot{\boldsymbol{\varepsilon}}_{\mu}^{(i)} & -\hat{\mathbf{A}}^{(i)}\left(\boldsymbol{\sigma}^{(i)}, \boldsymbol{\varepsilon}_{\mu}^{(i)}, t\right) \dot{\boldsymbol{\sigma}}^{(i)}=\hat{\mathbf{C}}_{\mu}^{(i)}\left(\boldsymbol{\sigma}^{(i)}, \boldsymbol{\varepsilon}_{\mu}^{(i)}, t\right) \quad(\mu=1, \ldots, n ; i=1, \ldots, N) \\
\sum_{\mu} \dot{\boldsymbol{\varepsilon}}_{\mu}^{(i)}= & \mathbf{b}^{(i)}\left\{\sum_{i} \mathbf{b}^{(i)^{\mathrm{T}}}\left[\sum_{\mu} \hat{\mathbf{A}}_{\mu}^{(i)}\left(\boldsymbol{\sigma}^{(i)}, \boldsymbol{\varepsilon}_{\mu}^{(i)}, t\right)\right]^{-1} \mathbf{b}^{(i)} V^{(i)}\right\}^{-1}\{\dot{\mathbf{F}}(t) \\
& \left.+\sum_{i} \mathbf{b}^{(i)^{\mathrm{T}}}\left[\sum_{\mu} \hat{\mathbf{A}}_{\mu}^{(i)}\left(\boldsymbol{\sigma}^{(i)}, \boldsymbol{\varepsilon}_{\mu}^{(i)}, t\right)\right]^{-1}\left[\sum_{\mu} \hat{\mathbf{C}}_{\mu}^{(i)}\left(\boldsymbol{\sigma}^{(i)}, \boldsymbol{\varepsilon}_{\mu}^{(i)}, t\right)\right] V^{(i)}\right\} \quad(i=1, \ldots, N)
\end{aligned}
$$

The equilibrium equations (5) and geometric relations (2) are implied in equation (7b). Equation (7a) expresses the stress-strain law. The functions $\widehat{\mathbf{A}}_{\mu}^{(i)}$ and $\hat{\mathbf{C}}_{\mu}^{(i)}$ describe the material properties in the centroid of the $i$ th element.

Equations $(7 \mathrm{a}, \mathrm{b})$ represent a system of $6(n+1) N$ non-linear ordinary first-order differential equations for the unknown components of $\sigma^{(i)}$ and $\varepsilon_{\mu}^{(i)}$. For the sake of brevity it is convenient to combine $\boldsymbol{\sigma}^{(i)}$ and $\boldsymbol{\varepsilon}_{\mu}^{(i)}$ in one column matrix denoted by $\mathbf{X}$, namely

$$
\mathbf{X}^{\mathrm{T}}=\left(\boldsymbol{\sigma}^{(1)^{\mathrm{T}}}, \ldots, \boldsymbol{\sigma}^{(N)^{\mathrm{T}}}, \boldsymbol{\varepsilon}_{1}^{(1)^{\mathrm{T}}}, \ldots, \boldsymbol{\varepsilon}_{n}^{(1)^{\mathrm{T}}}, \ldots, \boldsymbol{\varepsilon}_{1}^{(N)^{\mathrm{T}}}, \ldots, \boldsymbol{\varepsilon}_{n}^{(N)^{\mathrm{T}}}\right)
$$


Furthermore, denote the right hand side of system $(7 \mathrm{a}, \mathrm{b})$ by $\mathbf{R}(\mathbf{X}, t)$, which is a column matrix function. The left hand side of system $(7 a, b)$ has the form $\mathbf{L}(\mathbf{X}, t) \dot{\mathbf{X}}$ where $\mathbf{L}$ is a square matrix function. Finally, denote the column matrix function $\mathbf{L}^{-1}(\mathbf{X}, t) \mathbf{R}(\mathbf{X}, t)$ by $\mathbf{f}(\mathbf{X}, t)$; note that $\mathbf{L}^{-1}$ exists as long as the structure is stable. The system $(7 \mathrm{a}, \mathrm{b})$ may thus be concisely written as follows*

$$
\dot{\mathbf{X}}=\mathbf{f}(\mathbf{X}, t)
$$

\section{NUMERICAL INTEGRATION}

Having formulated the problem in terms of equation (9), which is a first-order matrix differential equation in standard form, higher-order numerical methods, such as the second- or fourth-order Runge-Kutta methods or the predictor-corrector methods, ${ }^{12}$ can be applied. These methods will clearly converge faster and allow larger time steps to be used without numerical instability, as compared with the conventional Euler process. However, the economics in computer time has so far been demonstrated only for the second-order Runge-Kutta method, ${ }^{15}$ and research here is still continuing.

In any of the step-by-step numerical methods, equation (9) is replaced by a system of algebraic matrix equations that can be solved successively. Each of these equations can be regarded as a matrix formulation of a certain linear elasticity problem with initial strains. Examining their physical significance, any numerical method may be formulated as a sequence of linear elasticity problems, independent of the method adopted for the analysis of each of the elasticity problems..$^{15,16}$ This enables the extension of the step-by-step algorithms to finite elements with variable strains, as well as to other than finite elements methods, ${ }^{16}$ which may sometimes be of advantage, as has been demonstrated in Reference 15 where the finite difference method was applied for bending of a rectangular plate in each time step.

\section{REFERENCES}

1. O. C. Zienkiewicz and Y. K. Cheung, The Finite Element Method in Structural and Continuum Mechanics, McGraw-Hill, New York, 1967.

2. R. W. Clough, 'The finite element method in structural mechanics', in Stress Analysis (Ed. O. C. Zienkiewicz and G. S. Hollister), Wiley, New York, 1965, Chap. 7.

3. A. Mendelson, H. H. Kirschberg and S. S. Manson, 'A general approach to the practical solution of creep problems', Trans. Am. Soc. mech. Engrs, Ser. D, J. bas. Engng, 81, 585-598 (1959).

4. E. L. Wilson, Finite Element Analysis of Two-dimensional Structures, Structural Engineering and Structural Mechanics, Report No. 63-2, University of California, Berkeley, 1963.

5. J. H. Argyris, 'Continua and discontinua', Proc. Conf. Matrix Meth. Struct. Mech., Wright-Patterson Air Force Base, Dayton, Ohio, October, 26-28 (1965).

6. C. A. Felippa, Refined Finite Element Analysis of Linear and Nonlinear Two Dimensional Structures, Ph.D. Thesis, Report No. 66-22, Division of Structural Engineering and Structural Mechanics, University of California, Berkeley, 1966.

7. R. S. Sandhu, E. L. Wilson and J. M. Raphael, Two Dimensional Stress Analysis with Incremental Construction and Creep, Report 67-34, Structural Engineering Laboratory, University of California, Berkeley, 1967.

8. G. A. Greenbaum and M. F. Rubinstein, 'Creep analysis of axi-symmetric bodies using finite elements', Nucl. Engng Des. 7, 379-397 (1968).

9. P. V. Marcal and I. P. King, 'Elastic-plastic analysis of two dimensional stress systems by the finite element method', Int. J. mech. Sci. 9, 143-155 (1967).

10. O. C. Zienkiewicz, M. Watson and I. P. King, 'A numerical method of viscoelastic stress analysis', Int. J. mech. Sci. 10, 807-827 (1968).

11. O. C. Zienkiewicz and S. Valliappan, 'Analysis of real structures for creep, plasticity and other complex constitutive laws', Conf. Mater. civ. Engng, University of Southampton, 1969 (Wiley, 1970).

12. A. Ralston, A First Course in Numerical Analysis, McGraw-Hill, New York, 1965.

* In the case of incremental elasto-plasticity, the matrix differential equation may be formulated only with stresses $\sigma^{(i)}$ as variables. This has been shown by Tocher and Varanasi. ${ }^{14}$ 
13. Z. P. Bažant, Approximate Methods of Analysis of Creep and Shrinkage of Nonhomogeneous Structures and Use of Computers (in Czech), Stavebnícky Casopis SAV (Bratislava), 1964; Concrete Creep in Structural Analysis (in Czech), SNTL (State Publishing House of Technical Literature), Prague, 1966.

14. J. L. Tocher and S. R. Varanasi, 'Finite element analysis of plasticity problems', Proc. Am. Soc. civ. Engrs, Joint Speciality Conference on Optimization and Nonlinear Problems, Chicago, 1968, pp. 180-186.

15. Z. P. Bažant, 'Numerical solution of nonlinear creep problems with application to plates', Int. J. Solids Struct. 7, 83-97 (1971).

16. Z. P. Bažant, 'Linear creep solved by a succession of generalized thermo-elasticity problems', Acta tech. Praha, 5, 581-594 (1967). 